\title{
High energy dissipative Raman soliton laser through XPM stabilization
}

\author{
Emre Ergeçen $^{1}$, Ugur Tegin $^{2}$, Parviz Elahi $^{2}$, Çagri Şenel $^{3}$ and F. Ömer Ilday ${ }^{2,4}$ \\ ${ }^{1}$ Department of Electrical and Electronics Engineering, Middle East Technical University, 06800 Ankara,, Turkey \\ ${ }^{2}$ Department of Physics, Bilkent University, 06800 Ankara,, Turkey \\ ${ }^{3}$ TUBITAK National Metrology Institute (UME), PO 54, TR-41470, Gebze, Kocaeli, Turkey \\ ${ }^{4}$ Department of Electrical and Electronics Engineering, Bilkent University, 06800 Ankara, Turkey
}

\begin{abstract}
Dispersion, nonlinearity and gain determine the intracavity pulse behaviour. We show that pump depletion and XPM play a significant role in the stabilization of high energy dissipative Raman solitons. Using this theoretical knowledge, we predict and demonstrate 7-nJ femtosecond pulses at $1120 \mathrm{~nm}$.
\end{abstract}

OCIS codes: (060.2320) Fiber optics amplifiers and oscillators; (140.3550) Lasers, Raman; (140.7090) Ultrafast lasers.

\section{Model}

Stable pulses in a cavity arise from the balance between competing mechanisms. Here, we report a novel synchronously pumped Raman laser generating linearly chirped Raman dissipative solitons. In our cavity, the stability of Stokes pulses is guaranteed by pump depletion and intracavity filtering.

In a synchronously pumped Raman cavity, Stokes signal emerges from spontaneous Raman scattering noise. The Stokes signal is amplified each roundtrip by the synchronized pump pulse supplied by the seed source. The gain experienced by the Stokes pulse is proportional to the instantaneous intensity of the pump pulse. Therefore, assuming no pump depletion, Raman gain behaves as a temporal gating similar to saturable absorption. Furthermore, due to the finite bandwidth of Raman response, Raman gain behaves as a spectral filter on the Stokes pulse. The terms related to the temporal and spectral filtering experienced by the Stokes pulse can be derived from the governing equation [1]:

$$
\frac{\partial A_{s}}{\partial z}+d \frac{\partial A_{s}}{\partial t}+i \frac{\beta_{2 s}}{2} \frac{\partial^{2} A_{s}}{\partial t^{2}}+\frac{\alpha_{s}}{2} A_{s}-i \gamma_{s}\left|A_{s}\right|^{2} A_{s}=g_{R}\left|A_{p}\right|^{2} A_{s}+\beta_{R}\left|A_{p}\right|^{2} \frac{\partial^{2} A_{s}}{\partial t^{2}}+i\left(2-f_{R}\right) \gamma_{s}\left|A_{p}\right|^{2} A_{s}
$$

where $g_{R}$ is the Raman gain and $\beta_{R}$ is the filtering term due to Raman gain bandwidth. In addition to stimulated Raman scattering (SRS), pump and Stokes pulses can interact via instantaneous Kerr effect.

Cross phase modulation (XPM) can strongly affect the spectral features and pulse shape of Stokes pulse. [2] Here, we show that XPM can also affect the stability of the copropagating pump - Stokes pulse pair assuming no pump depletion. In normal dispersion regime, the synchronisation between the pump and Stokes pulse is not immune to small perturbations, such as timing jitter in synchronization. To illustrate this instability, we utilize the semi-analytical technique called Method of Moments (MoM). MoM technique yields the evolution of pulse parameters such as pulse duration, chirp, peak intensity for given a priori pulse shapes. [3] After ignoring the SRS related terms and normalizing the governing equation, we obtain the following equation:

$$
i \frac{\partial A}{\partial z}+\frac{D}{2} \frac{\partial^{2} A}{\partial t^{2}}+|A|^{2} A=-v f(t) A
$$

where $\mathrm{D}$ is the normalized dispersion parameter (-1 if normal), $v$ is the non-negative XPM parameter and $f(t)$ is the normalized pump intensity approximated as $f(t)=\exp \left(-\frac{t^{2}}{\tau^{2}}\right)$. For no walk-off and depletion, the pump pulse $f(t)$ remains the same during the propagation. The most general form of the Stokes pulse shape can be taken as chirped Gaussian pulse $A(z, t)=A \exp \left(-(t-T)^{2} / \omega^{2}\right) \exp \left(i b(t-T)+i c(t-T)^{2}\right)$.

To show the XPM related instability between the pump and Stokes pulse, we will focus on the evolution of parameters $\mathrm{b}$ and $\mathrm{T}$. The differential equations governing their evolution obtained from MoM reads:

$$
\frac{d b}{d z}=-\frac{4 \sqrt{2} e^{-\frac{2 T^{2}}{2 \tau^{2}+\omega^{2}}} \omega v T}{\left(2 \tau^{2}+\omega^{2}\right)^{\frac{3}{2}}}
$$




$$
\frac{d T}{d z}=D b
$$

For an infinitesimal negative timing jitter in synchronisation $(b=0, T=-\varepsilon), b$ starts to increase. Increase in $b$ will result in a decrease in $\mathrm{T}$ in normal dispersion regime. Since an infinitesimal perturbation to time delay between Stokes and pump pulses grows without damping, the time delay between pump and Stokes is not immune to perturbations, rendering the equilibrium point unstable.

The destabilising effect of XPM can be alleviated by the pump depletion which is ignored in our previous analysis. As the Stokes pulse propagates, the synchronized copropagating pump pulse transfers energy to the Stokes pulse through SRS and gets depleted. Since the amount of depletion due to SRS is proportional to the Stokes intensities, we expect pump depletion occurs more at intensity maxima of the pump pulse. Therefore, the pump pulse collapses during propagation and gives rise to an intensity dip near the center of pump pulse where the Stokes pulse can stably propagate.
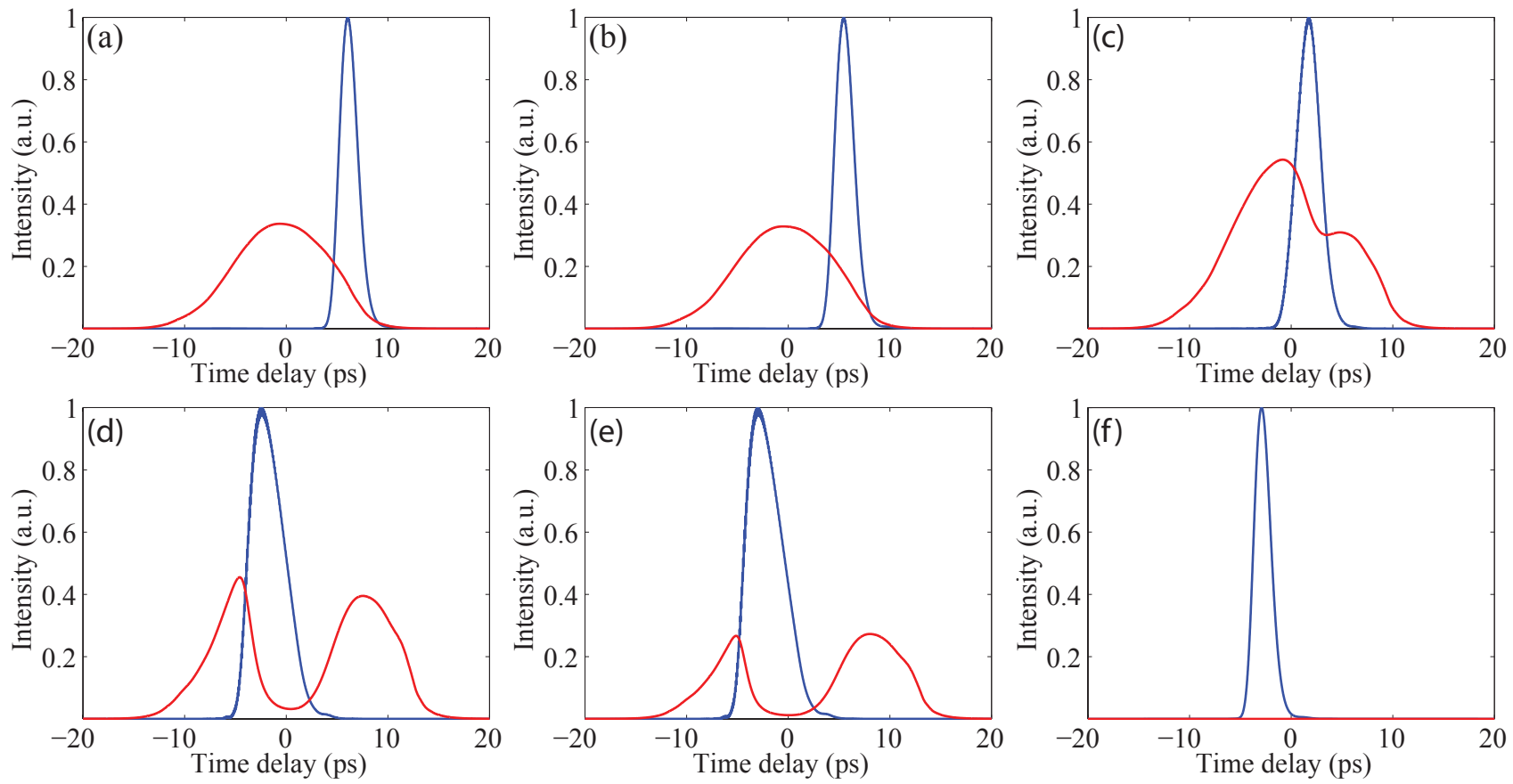

Fig. 1. Evolution of pump (red) and Stokes (blue) pulses (a) After synchronisation, (b) After $70 \mathrm{~cm}$ of SMF (c) After $1.5 \mathrm{~m}$ of gain segment (d) After $3.5 \mathrm{~m}$ of gain segment (e) After $80 \mathrm{~cm}$ of SMF (f) After the filtering stage

To test our theoretical predictions, we solve numerically the nonlinear Schrodinger equation with Raman, shock and third order dispersion terms. The simulated setup comprises $1.8 \mathrm{~m}$ of passive single mode fiber (SMF) and $3.5 \mathrm{~m}$ of $\mathrm{Yb}$ doped gain segment pumped with a $150 \mathrm{~mW}$ pump. After the Stokes and pump pulses are synchronized, they propagate $70 \mathrm{~cm}$ in passive fiber segment before entering the gain segment. Since the Stokes signal is amplified mostly in the gain segment, we introduce an initial time delay of $7.5 \mathrm{ps}$ between the pump and Stokes pulses to counteract walk-off and to ensure synchronization before the gain segment. After the gain segment, the pump - Stokes pulse pair propagates $80 \mathrm{~cm}$ in passive fiber segment and filtered by a Gaussian filter centered at $1120 \mathrm{~nm}$ with a bandwidth of $9 \mathrm{~nm}$. After the Gaussian filter, the Stokes pulse completes the roundtrip whereas the pump pulse is filtered out. The numerical results given in Fig. 1. are in accord with our theoretical expectations.

\section{Experimental Results}

Based on the aforementioned theory and numerical results, we experimentally developed a fiber Raman laser as shown in Fig.2. The setup consists of a pump laser source generating highly positive chirped pulses centered at 1065 $\mathrm{nm}$ and Raman oscillator. The chirped input pulses are led into the polarization-maintaining Yb-doped fiber amplifier and amplified to $760 \mathrm{~mW}$ average power (without feeding the output back into the amplifier) to generate the first 
Stokes wave. Next, we arrange intracavity Raman feedback with grating based filtering section while filtering out the pump. We use a translation stage to arrange to synchronize and temporally overlap the seed pulses at $1065 \mathrm{~nm}$ and the Stokes wave at $1120 \mathrm{~nm}$ precisely, which is confirmed by time-domain measurements.

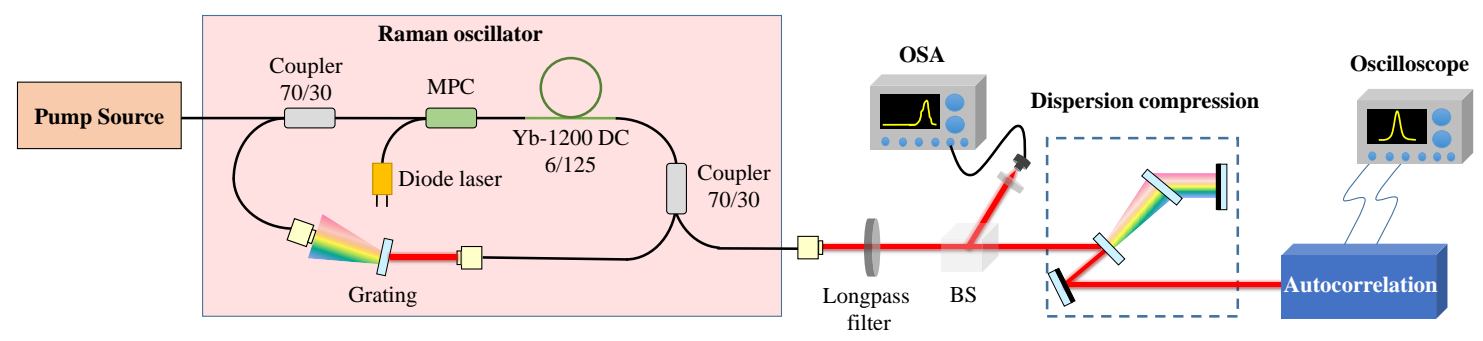

Fig. 2. Experimental setup. MPC, multi-pump signal combiner; DC, double clad; BS, beam splitter

When temporal pump-Raman overlap is achieved, the amplified spectrum is modified dramatically, with most of the energy being transferred to the Stokes wave at $1120 \mathrm{~nm}$ with $22 \mathrm{~nm}$ spectral width (Fig. 3.a insert). The pulses are compressed to $136 \mathrm{fs}$ with a diffraction grating pair(Fig. 3.a). For pump depletion, instead of a longpass filter at output segment we use a shortpass filter and suppress the Stokes components of the spectrum. Thus we obtain the pump pulse while Raman cavity is functional and measure the autocorrealtion of uncompressed pump pulse. The autocorrelation trace (Fig. 3.b) has similar features with a depleted Gaussian pulse.
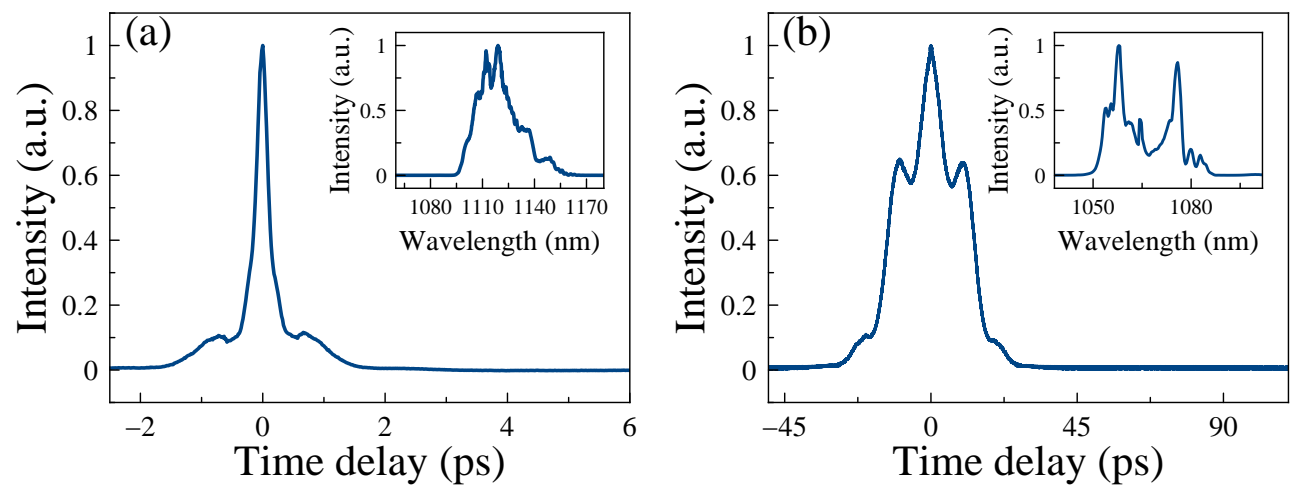

Fig. 3. (a) Measured autocorrelation trace of the dechirped pulse and the insert is measured Stokes output spectrum after longpass filtering (b) Measured spectra of pump pulse while Raman laser is functional and the insert is measured autocorrelation trace of the dechirped pump pulse

\section{References}

1. C. Headley III, G. P. Agrawal "Unified description of ultrafast stimulated Raman scattering in optical fibers," J. Opt. Soc. Am. B 13, 2170-2177 (1996).

2. R. R. Alfano, P. L. Baldeck, P. P. Ho, G. P. Agrawal "Cross-phase modulation and induced focusing due to optical nonlinearities in optical fibers and bulk materials," J. Opt. Soc. Am. B 6, 824-829 (1989).

3. E. N. Tsoy, A. Ankiewicz, N. Akhmediev "Dynamical models for dissipative localized waves of the complex Ginzburg-Landau equation,” Phys. Rev. E 73, 036621 (2006). 